\title{
Eltrombopag Managed Severe Immune Thrombocytopenic Purpura in Pregnancy: A Case Report, Latifa Hospital, DHA, Dubai, UAE
}

\author{
Komal Sundeep Hazari ${ }^{1}$, Atif Bashir E. Fazari ${ }^{1,2 *}$, Taghrid Gergawi ${ }^{1}$, \\ Hasan Mohamed A. Aal-Yaseen ${ }^{3}$, Nighat Aftab ${ }^{1}$ \\ ${ }^{1}$ Department of Obstetrics \& Gynecology, Latifa Hospital, DHA, Dubai, UAE \\ ${ }^{2}$ Faculty of Medicine, University of Medical Sciences \& Technology, Khartoum, Sudan \\ ${ }^{3}$ Department of Medicine, Clinical Haematology, Dubai Hospital, DHA, Dubai, UAE \\ Email: *atiffazari@hotmail.co.uk
}

How to cite this paper: Hazari, K.S., Fazari, A.B.E., Gergawi, T., Aal-Yaseen, H.M.A. and Aftab, N. (2019) Eltrombopag Managed Severe Immune Thrombocytopenic Purpura in Pregnancy: A Case Report, Latifa Hospital, DHA, Dubai, UAE. Open Journal of Obstetrics and Gynecology, 9, 991-996. https://doi.org/10.4236/ojog.2019.97096

Received: June 13, 2019

Accepted: July 15, 2019

Published: July 18, 2019

Copyright $\odot 2019$ by author(s) and Scientific Research Publishing Inc. This work is licensed under the Creative Commons Attribution International License (CC BY 4.0).

http://creativecommons.org/licenses/by/4.0/

\begin{abstract}
Immune thrombocytopenic purpura (ITP) is an acquired autoimmune disorder, defined by a platelet count of less than $100 \times 10^{9} / \mathrm{L}$, secondary to impaired production and immune destruction of platelets. Bleeding tendency is the main presentation of this condition. Clinical symptoms and investigations will confirm the diagnosis. Steroid is the first line of treatment. Although Rituximab and Thrombopoietin receptor agonists are useful second line agents in non-pregnant adults, the data about their role in pregnancy are still limited. We present the case of a 30 year old primigravida, who was a known case of chronic ITP since childhood; the course of her disease was fluctuating, for which oral steroids were used accordingly. She presented with gum bleeding and petechial rash with very low platelets count. She was sponsored by the Patient Support Program and was given Eltrombopag during the third trimester. She responded well to Eltrombopag with no noticeable side effects, neither to the mother nor to the baby so far. Eltrombopag has been assigned Category $\mathrm{C}$ by the Federal Drugs Agency (FDA) nevertheless there are no well controlled data in the literature about its role in pregnancy.
\end{abstract}

\section{Keywords}

Immune Thrombocytopenic Purpura, Eltrombopag, Pregnancy

\section{Introduction}

Idiopathic thrombocytopenic purpura (ITP), also known as Primary immune thrombocytopenic purpura and autoimmune thrombocytopenic purpura, is 
primarily a disease of increased peripheral platelet destruction, with most patients having IgG antibodies produced by patients B-cells and directed against specific platelet membrane glycoproteins GP IIb/IIIA. Autoreactive cytotoxic T cells along with humoral and cellular auto-immunity affect the megakaryocytes, also impairing platelet production [1].

ITP can present with spontaneous epistaxis, gum bleeding, petechiae and ecchymosis. ITP is diagnosed more commonly during pregnancy when they are being routinely screened for anemia in pregnancy. Isolated thrombocytopenia is the characteristic finding on complete blood count evaluation. Peripheral blood smear shows normal morphology of red blood cells, white blood cells and platelets, including large and clumped platelets. Manual platelet count using citrate anticoagulated blood is a more reliable indicator of the actual platelet counts as Ethylene DiamineTetraAcetic acid could cause platelet clumping. ITP should be considered in pregnancy when the platelet counts fall below 100,000/ul. It can be differentiated from Gestational thrombocytopenia, which is a close differential diagnosis, as the platelet counts usually don't fall below 100,000 in the latter. Other causes low platelets being the pregnancy-associated microangiopathic syndromes. Severe ITP should be considered when the platelet count falls below $50,000 /$ ul with bleeding symptoms requiring immediate therapy. Though the overall risk of bleeding in ITP is low and there is a weak correlation between the platelet counts and bleeding tendencies, risk of bleeding is high with platelet count of less than 10,000 and treatment aims at preventing potentially fatal intracranial and gastrointestinal bleeding in the mother [2].

The incidence of ITP varies from 1 to 5 per 100,000 adults in the general population [3]. Immune thrombocytopenia (ITP) occurs in approximately 1 to 3 in 1000 pregnancies [4]; approximately ten-fold higher compared to the general population. The estimated incidence of severe ITP in pregnancy is 0.83 per 10,000 maternities [5].

The approach to treatment of ITP in pregnancy depends on whether the patient is actively bleeding and the gestational age. Corticosteroids are the first drugs of choice if the patient has no major bleeding, while platelet transfusions and Intravenous immunoglobulins are preferred during active bleeding, unplanned delivery and Neuraxial anesthesia.

Thrombopoietin receptor agonists activate the platelet thrombopoietin receptor thus stimulating production of platelets and other hematopoietic cells. The data concerning safety o Thrombopoietin receptor agonists during pregnancy are limited, although they have been used effectively in the non-pregnant population.

We present a case of severe ITP who was successfully treated with Eltrombopag in pregnancy.

\section{Case Report}

A 35-years-old primigravida was referred to us at 18 weeks of gestation with complains of gum bleeding and petechiae over the legs. She was a diagnosed case 
of Chronic ITP since the age of 4 years. She had been on low dose steroids since then which was adjusted according to her platelet counts. Three years back, she had an episode of severe menorrhagia when her platelet dropped to 7000, and she was treated with steroids and platelet transfusions.

Her platelet count was found to be 6000 , with a manual platelet count of 19,000 . She was vitally stable. In view of the severe thrombocytopenia, she has advised admission for Intravenous steroids and monitored regularly with $\mathrm{CBC}$ and was discharged home on low dose oral steroids with regular followup. At 26 weeks, she stopped steroids due to mild muscle pains, but was reassured and advised to continue taking the minimal effective dose.

On follow up at 34 weeks, she still had complained of gum bleeding, her platelet count was 13,000. She was then commenced on Eltrombopag $50 \mathrm{mg}$ daily, under the sponsorship of "The Patient support Group program". She was booked for Elective Caesarean section at 38 weeks during the Multidisciplinary (MDT) meeting where in the consultant Obstetrician, Physician, Anesthetist, Neonatologist, Hematologist and Pharmacist were present. She showed a dramatic rise in her platelet count which peaked at 38 weeks to $240 \times 10^{9} / \mathrm{L}$ when she was delivered by Elective Caesarean section as advised by the MDT to avoid the bleeding risk (Figure 1).

She delivered a healthy female baby, weighing $2.5 \mathrm{~kg}$. She did not require any blood transfusion or platelet transfusion during the surgery. The postpartum and peripartum period was uneventful. The baby's platelet count was $237 \times 10^{9} / \mathrm{L}$ at birth as well on follow up showed normal range of platelets count. Both mother and her baby were discharged in a stable clinical condition. Patient was advised to stop Eltrombopag and follow up after 1 week. Postpartum at 1 week her platelet count dropped to $13 \times 10^{9} / \mathrm{L}$. To date both mother and child are healthy.

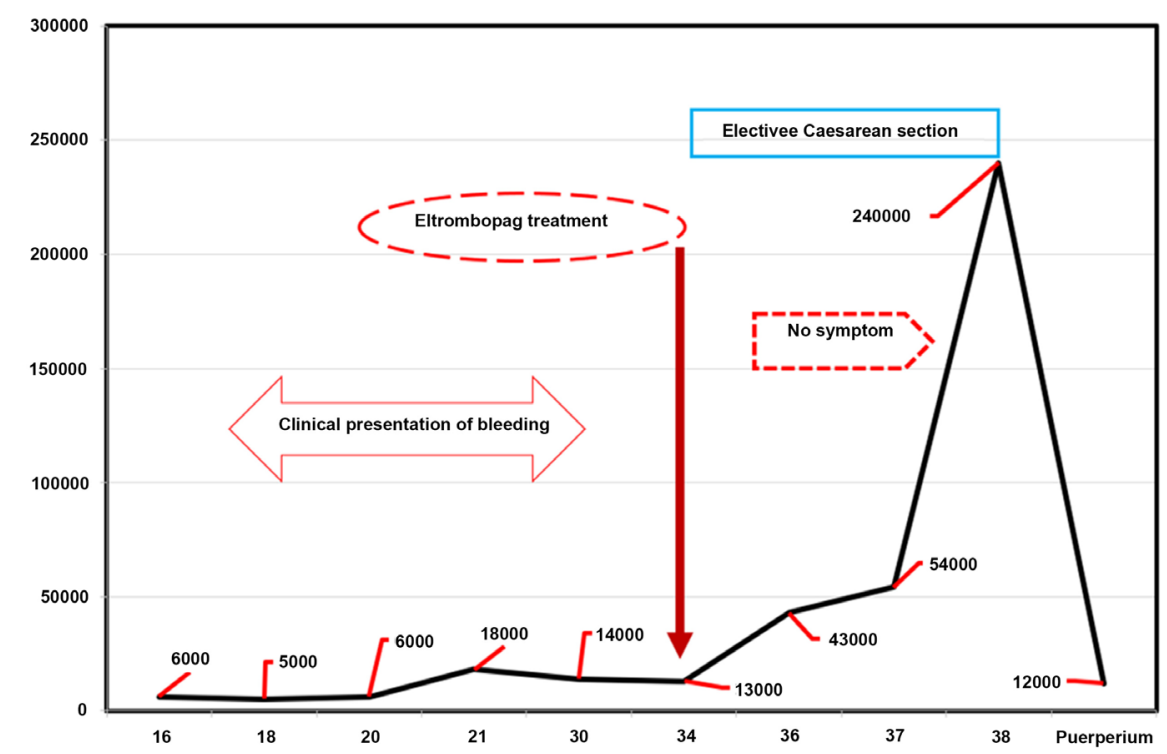

Figure 1. Shows the platelets count at presentation, Eltrombopag treatment and early puerperium. 
The baby was followed up regularly, at present she is 20 months old with normal milestones and growth.

\section{Discussion}

ITP is an autoimmune disorder caused by autoantibodies known as Platelet associated immunoglobulin (PAIg) directed against glycoprotein GPIIb/IIIa or GP $\mathrm{Ib} / \mathrm{IX}$ complex and other glycoprotein (GP) complexes. CD4-positive helper cells in reaction to platelet surface glycoproteins which possibly involves CD40/CD40L co stimulation produce antibodies leading to the autoimmune destruction.

These antibody coated platelets are destroyed by the tissue macrophages located in the spleen leading to their reduced life span. Both humoral and cellular immunity targeted at megakaryocytes, in addition to the autoreactive cytotoxic $\mathrm{T}$ cells leads to reduced platelet production [2].

The most common condition which can be confused with ITP is Gestational thrombocytopenia, which occurs in $4 \%-8 \%$ of pregnancies. The platelet count in gestational thrombocytopenia usually ranges between $100-150 \times 10^{9} / \mathrm{L}$. However, in $1 \%$ of pregnant women with gestational thrombocytopenia the platelet count ranges from $70-100 \times 10^{9} / \mathrm{L}$. These patients usually present during late pregnancy or delivery and it has no serious implications either to the mother or neonate. Other causes include the microangiopathic syndromes, namely: pre-eclampsia with HELLP syndrome, Thrombotic ThrombocytopenicPurpura, Hemolytic Uremic Syndrome and acute fatty liver of pregnancy [2].

Management of ITP during pregnancy depends on the platelet count, bleeding symptoms and the need of Neuraxial anesthesia or operative delivery. The aim of treatment is to reach a safe platelet count and prevention of severe gastrointestinal or intracranial bleeding, rather than reach a normal platelet count.

Maternal hemorrhage during delivery and severe neonatal thrombocytopenia leading to intracranial or visceral hemorrhage are of serious concern.

In the absence of bleeding, the platelet count threshold for vaginal delivery is $30 \times 10^{9} / \mathrm{L}$ and $50 \times 10^{9} / \mathrm{L}$ for Caesarean delivery. Platelet transfusion is indicated if platelet count is less than $10 \times 10^{9} / \mathrm{L}$ at any time, less than $30 \times 10^{9} / \mathrm{L}$ in the third trimester or if there are any signs of bleeding [1].

Glucocorticoids are the first line of therapy if patient has no life-threatening bleeding, as they are cost effective and non-teratogenic, compared to cytotoxic drugs like azathioprine and cyclophosphamide. In cases of life-threatening bleeding or during emergencies, Intravenous immunoglobulin can be considered safe therapy. Its effect is, however, transient and its cost is high.

The development of various growth factors in the field of Hematology has positively affected the treatment practice. After the era of Erythroid and myeloid growth factors which succeeded in stimulation of erythrocytes and neutrophils, we now have effective thrombopoietic growth factors available which can stimulate platelet production.

Thrombopoietin (TPO) is the major physiological regulator of platelet pro- 
duction, being produced by the liver and cleared by the TPO receptors on the platelets. Eltrombopag is an oral non peptide TPO receptor agonist which is highly effective in the treatment of refractory ITP and hepatitis $\mathrm{C}$ induced thrombocytopenia. It networks with the transmembrane domain of the TPO receptor ( $\mathrm{cMpl}$ ) causing enhanced platelet production, and also stimulates megakaryocyte growth and differentiation. After oral administration, the platelet counts start to rise by day 6 and attain peak levels after $12-15$ days.

Headache is a well-known complication of this group of drugs, other long-term risks include thrombosis, increased bone marrow reticulin and blast production. Rebound thrombocytopenia is frequentlyseen after discontinuation of therapy. The starting dose is $50 \mathrm{mg}$ daily, with a maximum dose of $75 \mathrm{mg}$ daily, and a reduced dose of $25 \mathrm{mg}$ daily in cases with hepatic impairment [6] [7].

Eltrombopag has been categorized as category C by the FDA. Due to lack of enough and well controlled studies in pregnancy, it should be used in pregnancy only if benefit outweighs the risks. Evidence of embryo lethality \& decreased fetal weight was observed in animal reproduction and developmental toxicity studies [1]. In our patient it was started in the third trimester, so there were no chances of embryo lethality.

It is not known if Eltrombopag is present in breast milk. Due to the potential for serious adverse effects in the breastfed infant, breastfeeding is not recommended by the manufacturer.

\section{Conclusion}

Eltrombopag can be considered as a safe treatment option in pregnant patients with severe ITP, who fail to respond to other therapies like steroids and immunosuppressant. The limiting factor is high cost of the drug and lack of studies in the pregnant population.

\section{Acknowledgements}

We would like to extend our thanks for team shared this case care and management. We appreciate Dr. Mahmoud Elhalik the neonatologist at Latifa hospital for his effort in following the neonate and his care.

\section{Ethical Approval}

Informed consent obtained from the case herself with agreement of Obstetrics\& Gynecology department for publication with confidentiality.

\section{Conflicts of Interest}

The authors declare no conflicts of interest.

\section{References}

[1] Purushothaman, J., Puthumana, K.J., Kumar, A., Innah, S.J. and Gilvaz, S. (2016) A Case of Refractory Immune Thrombocytopenia in Pregnancy Managed with Elth- 
rombopag. Asian Journal of Transfusion Science, 10, 155-158.

https://doi.org/10.4103/0973-6247.177204

[2] George, J.N. and Arnold, D.M. (2014) Immune Thrombocytopenia (ITP) in Adults: Clinical Manifestations and Diagnosis. UpToDate Inc., Waltham, MA.

[3] Terrell, D.R., Beebe, L.A., Vesely, S.K., Neas, B.R., Segal, J.B. and George, J.N. (2010) The Incidence of Immune Thrombocytopenic Purpura in Children and Adults: A Critical Review of Published Reports. American Journal of Hematology, 85, 174-180. https://doi.org/10.1002/ajh.21616

[4] Ferreira, I.J., Sousa, F., Vasco, E.M., de Amaral Areia, A.L., Moura, J.P., Carda, J., Ribeiro, L. (2018) Severe Immune Thrombocytopenia in Pregnancy Treated with Eltrombopag-A Case Report. Journal of Gynecology Obstetrics and Human Reproduction, 47, 405-408. https://doi.org/10.1016/j.jogoh.2018.06.010

[5] Care, A., Pavord, S., Knight, M. and Alfirevic, Z. (2018) Severe Primary Autoimmune Thrombocytopenia in Pregnancy: A National Cohort Study. BJOG: An International Journal of Obstetrics \& Gynaecology, 125, 604-612. https://doi.org/10.1111/1471-0528.14697

[6] Kuter, D.J. (2009) Thrombopoietin and Thrombopoietin Mimetics in the Treatment of Thrombocytopenia. Annual Review of Medicine, 60, 193-206. https://doi.org/10.1146/annurev.med.60.042307.181154

[7] Kuter, D.J. (2013) The Biology of Thrombopoietin and Thrombopoietin Receptor Agonists. International Journal of Hematology, 98, 10-23.

https://doi.org/10.1007/s12185-013-1382-0 